\title{
La ética en la investigación: el caso con los tule (kunas) de Urabá, Colombia
}

\author{
Gloria Alcaraz ${ }^{1}$, Adriana Correa ${ }^{2}$
}

${ }^{1}$ Facultad de Enfermería, Universidad de Antioquia, Medellín, Colombia.
2 Facultad de Medicina, Universidad de Antioquia, Medellín, Colombia.

La investigación Cultura, alimentación y malaria entre los tule (kuna) se realizó en el resguardo de Caimán Nuevo, Urabá antioqueño, Colombia, y buscó analizar la prevalencia de la malaria y sus relaciones con la hipovitaminosis $\mathrm{A}$ y el estado nutricional, y comprender las concepciones y prácticas de esta etnia frente a la enfermedad.

El objetivo del ensayo es reflexionar sobre la aplicación de los principios éticos y los valores morales en el desarrollo de esta investigación, en la cual se hizo patente la diversidad cultural de los grupos: la cultura de los indígenas tule (kuna), con un sistema médico tradicional holístico, y la del grupo de investigación, centrada en el modelo biomédico y atada a la asignación aleatoria y a la consecución de una muestra representativa. Al margen de los dilemas que se presentaron al elaborar el proyecto, surgieron otros dilemas durante el trabajo de campo que exigieron tomar decisiones rápidas, para lo cual la academia nos prepara muy poco.

Se resalta la necesidad de que la reflexión ética permee todo el proceso investigativo en una relación de horizontalidad entre sujetos, en la cual la dignidad humana de los participantes y la unicidad de la cultura del colectivo debe estar por encima de los fines del proyecto.

Es urgente el establecimiento de comités de ética nacional y locales para preservar la vida y las culturas de los grupos étnicos, mucho más con las actuales políticas comerciales y de salud en un mundo globalizado.

Palabras clave: grupos étnicos, ética en investigación, medicina tradicional, investigación biomédica, comités de ética de la investigación, consentimiento informado.

\section{Research ethics: the case with the tule (kuna), Urabá, Colombia}

An ethical analysis was performed during the development of a research project titled "Culture, nourishment and malaria in the Kuna aboriginals of Urabá, Colombia." This project had 2 objectives; (1) to investigate the prevalence of malaria and its relationship with hypovitaminosis A and nutritional status, and (2) to understand the community's conceptions and practices towards malaria. The current paper summarizes reflections concerning the application of ethical principles and moral values during the course of the malaria research project. Two intercultural relationships were clearly defined: the culture of the Tule (Kuna) Indians, with a traditional medical system, and the culture of the research group, focused on the biomedical model. The rationale and the discussion proposed during the writing of the project proposal was presented as well as the dilemmas that developed during the project within the communities. These problems required rapid decision-making in situations for which the scientists had not been adequately prepared. A need was indicated for application of ethical principles to permeate the analysis throughout the research process in a horizontal manner, such that the human dignity of the participants and the ethnicity of the community are preserved above the purposes of the project. In the research process, mediation between the ethics of conviction and the ethics of consequences must take precedence, with both outcomes tied to an informed consent in place during the process. The establishment of national and local research ethics committees was indicated as necessary to preserve the lifestyle and culture of the ethnic groups. These values were seen as endangered given the trading pressures and health policies in a globalized world.

Keywords: Ethnic group, ethic research, traditional medicine, biomedical research, research ethics committees, informed consent. 
La investigación Cultura, alimentación y malaria en los indígenas kuna se realizó en el resguardo de Caimán Nuevo, localizado entre los municipios de Turbo y Necoclí del Urabá antioqueño, Colombia. Los tule (kuna) son oriundos de las cuencas de los ríos Atrato y Baudó (1). Están localizados principalmente en las islas de San Blas en Panamá (unos 200.000) (2), y un pequeño número en nuestro país (aproximadamente 1.500). Los tule (kuna) de Colombia están ubicados en dos resguardos, Makilakuntiwala (Arquía) en el Urabá chocoano, e Ipkikuntiwala (Caimán Nuevo) en el Urabá antioqueño.

El resguardo Ipkikuntiwala está dividido en tres sectores: alto, medio y bajo Caimán. La población asciende a 1.028 personas. La estructura social está fundamentada en la familia extensa matrilocal (3), cuya autoridad está ejercida por el sakka o jefe de la familia. La organización política está liderada por el saila (cacique o jefe principal), quien preside el onmaket (congreso); lo siguen los alkal (voceros de la comunidad), los soal ipeti (policías) y los sapin tummakan (fiscales) (4). El onmaket se celebra semanal o quincenalmente y a él asisten hombres, mujeres y niños; en medio de cantos y discursos se mantiene viva la cultura tule (kuna).

Según los kuna, el mundo fue creado por Páptumat y consta de 12 capas en las cuales se encuentran los espíritus de los animales, de los antepasados, de los héroes culturales y de las enfermedades (5). La enfermedad es vista como el producto de la interacción del grupo con el hábitat y del desequilibrio en el interior de la estructura social. Las enfermedades de filiación cultural son tratadas a través del sistema etnomédico kuna y las no culturales por el sistema médico occidental (6).

El sistema etnomédico está liderado por el nele (chamán), o médico tradicional; generalmente, este estatus se logra por adscripción (3); continúa en jerarquía el inatuledi, quien estudia la botánica

Correspondencia:

Gloria Alcaraz, Facultad de Enfermería, Universidad de Antioquia, apartado aéreo 1226, Medellín, Colombia.

Fax: 2110058

glory@tone.udea.edu.co

Recibido: 16/05/05; aceptado: 27/10/05 y los cantos para diagnosticar y curar enfermedades; el absogedi, quien previene la expansión de enfermedades y las $m u$, que son mujeres que atienden los partos. Existen también varios especialistas en cantos terapéuticos.

El sistema económico tradicional se basa en la agricultura, la caza y la pesca, y la cría de animales domésticos para el consumo. Los principales productos agrícolas son el maíz (Zea mays L.), el plátano (Musa paradisiaca L.), el arroz (Oriza saliva L.), el coco (Cocos nucifera L.), la yuca (Manihot esculenta Graz) y frutas de cosecha (4).

Los kuna prefieren ser llamados por su etnónimo tule, que significa "gente de oro", y cuando se les llama kuna, solicitan que se escriba con k; por tal motivo, a lo largo del artículo los llamaremos tule o kuna.

En el Urabá antioqueño la prevalencia de malaria es alta, tal como lo muestran las cifras del índice parasitario anual (IPA) entre 1998-2003 (Turbo con 26,85 y Necoclí con 10,93 por mil expuestos) (79); se desconoce la prevalencia en la comunidad kuna. La investigación buscó analizar por medio del método cuantitativo la prevalencia de la malaria y sus relaciones con la hipovitaminosis $\mathrm{A}$ y el estado nutricional, así como comprender, por medio del método cualitativo, las concepciones, actitudes y prácticas frente a la malaria en este grupo indígena.

Mientras que países como Canadá cuentan con una comisión de ética para orientar, regular y vigilar los trabajos e investigaciones que se realizan con indígenas, en Colombia carecemos de dichas disposiciones, razón por la cual las consideraciones éticas para la elaboración del proyecto se trabajaron con el apoyo de la resolución 008430 de 1993 del Ministerio de Salud de Colombia y la propuesta de Normas Éticas Internacionales para las Investigaciones Biomédicas con Sujetos Humanos propuestas por el Consejo de la Organización Internacional de las Ciencias Biomédicas (CIOMS) y la Organización Mundial de la Salud (OMS), y publicadas por la OPS/OMS en 1996 (10).

El objetivo de este artículo es reflexionar sobre la aplicación de los principios éticos, los valores morales y la responsabilidad en el desarrollo de 
la investigación. Al plantear un proyecto de este tipo surgen múltiples dilemas éticos que los investigadores tratan de resolver desde su conocimiento; sin embargo, cuando se llega al trabajo de campo, surgen otros más difíciles y complejos (11). En este texto se presentan los dilemas surgidos durante el desarrollo de la investigación con los kuna, así como las diferentes formas de dirimirlos. Los aciertos 0 desaciertos que mostramos en el texto pueden inducir a los investigadores o trabajadores de la salud a reflexionar sobre los principios éticos y su aplicación en trabajos con comunidades indígenas.

El texto se desarrolla en tres partes: la primera aborda las consideraciones éticas que se observaron para la concertación con la comunidad y la elaboración escrita del proyecto; la segunda desarrolla las reflexiones éticas y las decisiones tomadas durante la ejecución de la investigación, $y$, finalmente, se hace una reflexión sobre los principios éticos en las investigaciones con grupos étnicos.

\section{Concertación inicial con la comunidad, negociación de los temas y elaboración de la propuesta}

En febrero de 2002 la coordinadora de la investigación aceptó una invitación de la comunidad para asistir a una reunión cuyo objetivo era concertar entre ellos y personas no indígenas, proyectos de investigación y programas de intervención en salud, agropecuarios y de recuperación de territorios, entre otros. Para efecto de esta reunión, los sailas (jefes) del sector de Caimán bajo invitaron a los diferentes profesionales a un lereo (ritual de rezo y armonización con el cosmos y con el tule mismo), al que también asistió la comunidad indígena. En esta reunión, la investigadora expresó el deseo de adelantar trabajos relacionados con la salud. Ambas partes sugirieron algunos temas: cultura y salud, salud oral, nutrición, malaria, parasitismo intestinal y tuberculosis. Finalmente, se acordó investigar sobre cultura, alimentación y malaria. Para conocer el estado de la malaria, se hizo una búsqueda activa y de registro de casos en la zona; el estado nutricional se determinó mediante antropometría, el estado de la vitamina A por su cuantificación en sangre venosa, y el consumo de betacaroteno en la dieta a través de una encuesta alimentaria. Se indagó sobre las percepciones, conocimientos y prácticas de malaria en esta comunidad. Luego se negociaron las acciones de reciprocidad entre los tule (kuna) y el grupo de investigación y, por último, se propuso la elaboración del proyecto y la búsqueda de financiación.

Este estudio, entonces, se inició concertando la temática con la comunidad, acorde con la necesidad sentida y expresada por ellos, y tuvo en cuenta tres aspectos: el primero fue la experiencia de algunos de los miembros del grupo de investigación en salud con colectivos étnicos $(6,12,13)$ guiada por los planteamientos de Colombero (14) para desarrollar la sensibilidad de los investigadores hacia el otro y su unicidad, basada en el respeto y en la autonomía de las comunidades indígenas y de sus miembros en este caso. El segundo aspecto que se tuvo en cuenta fue el derecho constitucional de los indígenas en Colombia, plasmado en la Constitución Política de 1991, y las luchas de las organizaciones indígenas de Colombia que han abierto el espacio para visualizar, reconocer y respetar las particularidades culturales de cada pueblo indígena. Cada organización indígena y cada cabildo tienen la autonomía para permitir los trabajos en salud dentro de sus territorios y con sus miembros. El tercer aspecto tuvo que ver con la ejecución del proyecto: la concertación con la comunidad y la incorporación de jóvenes como traductores y, en parte como asistentes de investigación, permitió la apropiación, el apoyo y la colaboración de los tule en el desarrollo de la investigación.

La reciprocidad se evidenció en el aporte de los tule con sus conocimientos sobre lo que les es propio, sus concepciones frente al mundo, a la malaria, a los alimentos, así como a la toma de la muestra de sangre. El equipo de investigación ofreció sus conocimientos sobre salud, de acuerdo con su formación. La comunidad solicitó capacitar a una persona tule (kuna) en la toma, lectura, diagnóstico y tratamiento de la malaria, ya que tienen presente lo relativo de su sistema médico 
y son conscientes de que la curación de algunas enfermedades requiere del sistema biomédico, por lo que existe la preocupación de que miembros de su comunidad se preparen tanto en el sistema médico tule (kuna) como en el biomédico actual. En el diálogo con la comunidad se analizó y se discutió la reciprocidad teniendo en cuenta los principios éticos de beneficencia, de no maleficencia hacia los tule (kuna), y la cooperación mutua entre investigadores y comunidad (15).

Una vez concertados los temas de estudio y las acciones de reciprocidad se comenzó la elaboración del proyecto y la búsqueda de financiación.

Como ya se dijo, los aspectos ético-legales considerados en la propuesta de investigación se orientaron por la resolución 008430 de 1993 del Ministerio de Salud de Colombia, referida a las normas científicas, técnicas y administrativas para la investigación en salud. Para esta investigación eran relevantes el capítulo I, sobre los aspectos éticos de la investigación con seres humanos, y el capítulo II, sobre la investigación con comunidades; de allí se tomaron los elementos necesarios para la elaboración del consentimiento informado, la clasificación del riesgo y los compromisos que adquirieron los investigadores (16).

La propuesta de investigación señaló que se tendrían en cuenta, además de los principios éticos fundamentales de respeto por las personas, de justicia y de beneficencia - no maleficencia, la declaración de Helsinki de 1987 y la propuesta de Normas Éticas Internacionales para las Investigaciones Biomédicas con Sujetos Humanos, realizada por el Consejo de la Organización Internacional de las Ciencias Biomédicas (CIOMS) y la Organización Mundial de la Salud (OMS), publicadas por la OPS/OMS en 1996 (10).

La propuesta planteaba que a las personas o familias que desearan participar en la investigación se les daría a leer el consentimiento informado; en caso de no saber leer, un traductor lo haría; los investigadores se comprometían a explicar y aclarar las preguntas surgidas. En caso de que el jefe de familia aceptara y autorizara la participación de sus integrantes, en el consentimiento se anotaría el nombre de cada una de las personas representadas por el jefe de familia. Si las personas o familias aceptaban, firmarían el consentimiento, o colocarían su huella dactilar en caso de no saber firmar; igualmente, firmarían o pondrían sus huellas dactilares los dos testigos y el investigador. En cualquier momento el tule (kuna) podía retirarse del proyecto. Después de cada salida de campo, la investigadora principal debía hacer una evaluación del cumplimiento de los aspectos éticos. Con base en los resultados de las evaluaciones, se tomaban los correctivos correspondientes.

En cuanto a la metodología, el estudio contemplaba dos enfoques: el cualitativo, centrado en la perspectiva etnográfica, en la que los investigadores participan, abiertamente 0 de manera encubierta, en la vida de las personas durante un período de tiempo; se observa el fenómeno estudiado, se pregunta, se escucha, se participa de las actividades del grupo y se realizan entrevistas (17). Con el método etnográfico se busca dar sentido a las expresiones e informaciones ofrecidas por los participantes, es decir, comprender el modo de vida de un grupo desde el punto de vista de los integrantes de esa colectividad; en este caso, se buscaba conocer y comprender las concepciones, actitudes y prácticas culturales de los tule relativas a la malaria y a la alimentación.

El segundo enfoque, el cuantitativo, se empleó para conocer el estado de la malaria, la concentración de la vitamina $A$ en sangre y el estado nutricional. Al comenzar a aplicarlo se presentó un dilema ante la propuesta sobre la asignación aleatoria de las personas para conformar la muestra. Holmes et al. (18), en sus investigaciones con jóvenes aborígenes de Australia, señalaron las dificultades para la conformación de muestras aleatorias debido, entre otras cosas, a la falta de registros actualizados para seleccionar a las personas y a las argumentaciones políticas de los individuos para sustentar su participación en los estudios o no hacerlo.

En este estudio se calculó una muestra representativa de 265 personas para evaluar el 
estado nutricional por mediciones antropométricas, el consumo de beta-caroteno mediante encuestas alimentarias y la concentración sérica de vitamina A. Nuestro conocimiento de la cultura tule y el interés de los indígenas por dinamizar su identidad nos llevó a los siguientes interrogantes.

¿Cómo hacer la asignación aleatoria de los tule (kuna) para el estudio? ¿Cómo llegar a un compromiso entre la aplicación de la ética en la elaboración de investigaciones biomédicas con otras culturas, o durante su desarrollo, y el paradigma biomédico centrado en la asignación aleatoria?

La reflexión continua del grupo generó otras preguntas: ¿sería bien visto por la comunidad la selección de las personas o familias para el estudio? ¿Qué dificultades acarrearía esta selección? ¿Cómo operaría la selección con los códigos y normas culturales? ¿Se podrían aplicar reglas de la cultura occidental a la cultura tule (kuna)? Si no se hacía la asignación aleatoria, ¿de qué manera la epidemiología clínica evaluaría el trabajo en cuanto a la representatividad de los tule (kuna) que participaban en el estudio?

Los objetivos del estudio en su dimensión cuantitativa y los medios para lograrlos abocaron al equipo a una reflexión nutrida por el concepto de dignidad humana expresado por Kant, el cual considera a los seres humanos como fines por sí mismos y no solamente como medios, y en las orientaciones de la bioética, la responsabilidad de acatar el principio del respeto a los valores morales y la aplicación del principio de no maleficencia (19). Finalmente, el grupo de investigadores, al considerar que el número representativo de 265 personas no tendría lógica dentro de los códigos culturales de los tule, y que para esta comunidad no sería completamente comprensible la selección aleatoria de las personas para participar en el proyecto (puesto que se calificaría como discriminatorio y buscarían razones económicas, políticas o espirituales para explicar la inclusión o la exclusión de las personas, lo que generaría una atmósfera de inconformidad dentro del colectivo tule), tomó la siguiente decisión: "en la medida en que las personas o familias kuna acepten participar, harán parte de la muestra", y así fue plasmada en la propuesta de investigación.

En este sentido, el equipo de investigación se acogió al principio de respeto por la individualidad, es decir que dentro de un colectivo cada persona es diferente y se concibe como un interlocutor válido (20). Se acogió, igualmente, el principio de respeto por la unicidad, esto es, mirar holísticamente a una persona o cultura (21).

Articulado a éstos, también están los principios de los investigadores, que tienen que ver con la comunicación, la capacidad de escucha, la comprensión (14), la responsabilidad (15) y la competencia profesional (22). Dicha competencia permitió, frente al paradigma biomédico de la asignación aleatoria, inclinarse a favor de la cultura indígena tule (kuna) y tomar decisiones en este marco de respeto.

Elaborada la propuesta, el saila principal entregó una carta de compromiso con la cual apoyaba el proyecto; igualmente, la Organización Indígena de Antioquia dio el aval para su ejecución. Siguiendo los requisitos para la gestión de proyectos de investigación solicitados por el sistema de investigación de la Universidad de Antioquia y por Colciencias, la propuesta fue evaluada por personas expertas en el tema y por el Comité de Ética de la Universidad de Antioquia. Obtenida la aprobación y la financiación de estas dos entidades, se inició el desarrollo de la investigación.

\section{Aspectos ético-legales en el desarrollo del trabajo de campo}

Después de hacer los contactos con las direcciones locales de salud de los municipios de Turbo y Necoclí, se realizó el desplazamiento hacia el resguardo indígena, sector de Caimán bajo. Al llegar a la comunidad se hizo la presentación ante el saila con el fin de coordinar el trabajo. En el onmaketse presentó nuevamente la propuesta de investigación con sus objetivos y los métodos para obtenerlos. Se esperaba un aspirante para el curso sobre malaria, tal como se había acordado previamente, pero la comunidad había seleccionado a cinco jóvenes aduciendo la necesidad de preparar a varias personas para 
suplir la atención de los casos de malaria en los momentos en que el responsable del puesto de salud no estuviera. La expectativa de los cinco jóvenes frente a esta preparación fue muy importante, puesto que para ellos era un paso para cualificarse como promotores de salud o como auxiliares de enfermería.

El equipo de investigación, conformado por docentes y estudiantes de la Universidad de Antioquia, se vio obligado a tomar una decisión rápida frente a la comunidad reunida. De un lado, preparar cinco personas implicaba más inversión de tiempo y, de otro lado, estaba el principio de de igualdad y equidad: todos los jóvenes aspirantes reclamaban igualdad de oportunidades para la adquisición de conocimientos; por otra parte, con base en el principio de equidad (19), los investigadores debían aportar según sus capacidades y preparación a la formación de los tule (kuna). Se tomó entonces la decisión de preparar a los cinco aspirantes.

En esta fase se inició la búsqueda activa de malaria con la toma y lectura de gota gruesa; no se presentaron objeciones ni dificultades, puesto que el examen de malaria es común entre ellos por encontrarse en una área endémica; los miembros del grupo de investigación ofrecieron la atención a los enfermos de malaria y se inició la preparación de los microscopistas y, también así como también la indagación sobre las concepciones culturales y las implicaciones de la extracción de sangre venosa. Se inició, igualmente en esta primera fase, la familiarización entre la comunidad tule (kuna) y los investigadores y el intercambio de ambos saberes médicos, orientados por el principio de beneficencia - no maleficencia.

Finalizado el primer trabajo de campo, se fijó la fecha de regreso de los investigadores a la comunidad para continuar la investigación y la capacitación en malaria de los jóvenes.

En cuanto al segundo trabajo de campo, al llegar a la comunidad de alto Caimán se había concertado con los sailas nuestra presentación del día siguiente en el onmaket (congreso). Nuestra carta de entrada para obtener la simpatía común fue la culminación de la preparación en malaria de los cinco jóvenes tule (kuna) y su certificación.

Una de las decisiones en el onmaketfue que "cada familia participará en esta fase de la investigación". Los alkal (policías) se encargaron de enviar organizadamente a las familias que estaban en el congreso al puesto de salud (ubicado junto a la casa donde se realiza el onmaket), e incluso se nos llamó la atención por la "lentitud" en la atención. Esta llamada "lentitud" se debió al proceso de traducción del consentimiento de la lengua castellana a la tule, la comprensión, y la firma por parte de cada jefe de familia tule (kuna) del consentimiento informado, que buscaba hacer valer el principio de respeto a la autonomía, a la unicidad y a la individualidad (23).

A pesar de que el proyecto había sido discutido y aprobado previamente con los sailas, en el momento de hacerse efectiva la recolección de los datos de la investigación se analizó y se discutió nuevamente el consentimiento informado en el onmaket. Seguimos las recomendaciones dadas por diferentes autores: para Hammersley y Atkinson (17) las personas estudiadas por los investigadores sociales deben ser informadas en forma clara y detallada y su consentimiento debe ser incondicional. Munhall (24) se refiere a un "consentimiento en proceso" que significa negociar y cambiar las condiciones en la medida en que el proceso avanza o cuando se requiera. El Sistema de Salud Pública de Canadá cuenta con una mesa de trabajo (Institucional Review Board, IRB) (25) que guía a las personas para asegurar la aplicación de la ética en comunidades indígenas, y que contempla, entre otros, el consentimiento en proceso, argumentando que las comunidades tienen derecho a ejercer su soberanía y su autodeterminación.

Con los tule nuestras consideraciones fueron las siguientes: cada cultura tiene su forma de concebir el mundo, de verlo y vivenciarlo, tiene sus códigos de comunicación y sus normas. Muchas veces el discurso expresado, que parece muy claro, no lo es totalmente para la comunidad; por tanto, es necesario socializar la investigación y sus objetivos cada vez que se hace trabajo de campo 
y garantizar a los indígenas el derecho a continuar en el proceso investigativo o no hacerlo.

En nuestro caso, el consentimiento informado siguió el siguiente proceso: discusión en la asamblea general (onmaket), discusión con cada familia o persona en la primera fase (toma de gota gruesa, mediciones antropométricas y entrevistas); discusión y concertación en la asamblea general y en forma individual para la segunda fase de la investigación (toma de sangre venosa).

En la primera fase, el consentimiento fue analizado con los sailas y los médicos tradicionales; suscitó discusión la toma de sangre venosa para determinar los niveles de vitamina $A$, ya que si bien el tule (kuna) está familiarizado con la toma de sangre capilar del dedo para el diagnóstico de malaria, no lo está con la extracción de cinco centímetros cúbicos de sangre venosa. Frente a la extracción de sangre venosa resaltamos tres aspectos importantes, expresados en las siguientes citas:

- "Pasó un caso en San Blas (Panamá); con esa gente había una gringa que vivía con ellos hacía mucho tiempo. En la comunidad había una viejita, muy viejita y muy sana, la gringa se preguntaba: esta viejita con tantos ánimos y tan bien, ¿cómo hace? Un día la gringa le sacó un pelo de la coronilla de la cabeza, lo llevaron a un laboratorio y descubrieron un hueco en el pelo y que la señora tenía la contra de todas las enfermedades. Como antiguamente no se dejaban sacar sangre, pasó que la mujer gringa le hizo tomar un jarabe a la viejita para que se enfermara, tres días estuvo enferma, le sacaron sangre engañada, con esto confirmaron que tenía la contra de todas las enfermedades y que eso sólo estaba en la raza kuna" (líder de 70 años).

- $\quad$ "El indio no tiene nada de raro fisiológicamente, pero descubrieron que la sangre indígena servía para la diabetes, entonces en todos los hospitales nos daban carné para sacarnos sangre. Entonces nos dijeron que para sacar sangre, para vender sangre, no, esa cosa nos alertaron" (líder de 75 años).

- Consideran que el tule nace con varias energías o fuerzas espirituales: niga (energía que sólo se pierde con la muerte, es susceptible de fortalecer o debilitar), kurkin (sabiduría localizada en la cabeza, puede ser desplazada por un demonio) y purba (espíritus, almas, o doble espiritual); existe una sola purba, pero conformada por varias, entre ellas: purba de corazón, de sangre, de rodilla, de estómago, de saliva (26). La pérdida de una de las purbas pone a la persona en una situación de peligro y en riesgo de enfermar; las más peligrosas son la pérdida de purba de corazón o de sangre.

Se hizo evidente entonces la dificultad para evaluar los niveles séricos de vitamina $A$. Los símbolos del cuerpo hacen parte del almacén de símbolos que construye cada cultura, los orificios del cuerpo simbolizan los puntos especialmente vulnerables, de ellos sale material marginal como saliva, lágrimas, heces y sangre; transgredir las márgenes simbólicas del cuerpo constituye un peligro (27), extraer sangre al tule con una jeringa implica, en primer lugar, violentar las márgenes simbólicas del cuerpo y, en segundo lugar, el peligro de perder purba.

Por otra parte, permitir la extracción de sangre indica un regalo de purba para los investigadores; así, los tule necesitan sentir confianza de que el investigador "no someterá esta purba a un brujo malo que pueda hacer un daño al tule (kuna) o a la comunidad en general". Discutieron también que "el waga (no indígena) llega, lleva sangre, estudia el humanismo del tule, patenta y el tule se queda sin saber qué pasó".

Esta situación suscitó en el equipo la reflexión sobre los principios de respeto, de autonomía y de beneficencia-no maleficencia frente a la cultura tule y a sus integrantes. Después de explicar, utilizando como ejemplo un alimento clásico de ellos, que los investigadores sólo llevarían el suero (plasma) y no los glóbulos rojos (para ellos sangre), y que con el suero (plasma) sólo se estudiaría la vitamina $A$, los sailas aceptaron la extracción de sangre venosa, pero añadieron que "queda a voluntad de cada tule participar en esta parte del trabajo". Según Fry (15), ocurre aquí una transferencia de valores de la cultura tule a los valores individuales, comprendidos como los valores y actitudes que constituyen la base del 
comportamiento y determinan la forma en que cada persona experimenta la vida. Quedó claro ante estos líderes y ante la comunidad misma, que cada indígena tenía la libertad de decidir su participación en el estudio o de retirarse en el momento que considerara pertinente. Para el kuna, el consentimiento informado se tornó en una forma de validar el acuerdo obtenido entre la comunidad y el equipo de investigación.

En la asamblea también se hizo un informe sobre el estado de la malaria (muy alarmante por cierto), y se acordó con los líderes la fecha del próximo trabajo de campo, las actividades que se llevarían a cabo y la realización del taller de salud y alimentación.

En los trabajos de campo anteriores se había completado la muestra: la medición antropométrica, la búsqueda activa de malaria y las encuestas alimentarias estaban realizadas en el $100 \%$; las encuestas cualitativas estaban alcanzando la saturación, y sólo faltaba iniciar la toma de muestras para conocer el estado de la vitamina $A$. Los trabajos de campo siguientes tuvieron como objetivo la extracción de la sangre venosa.

En este grupo étnico las vitaminas son de gran importancia para fortalecer el cuerpo y las energías espirituales, para lo cual consumen alimentos específicos, se hacen baños y se ejecutan rituales especiales. Por tanto, durante la posterior salida de campo no se encontró objeción al objetivo de trabajo y, nuevamente, los tule le dieron al grupo de investigación un voto de confianza, aunque los medios para lograrlo implicaban riesgos para el individuo y la colectividad, sumado esto al miedo ante el dolor producido por la punción. Los sailas insistieron en la importancia de evaluar el estado de la vitamina A y apoyaron la realización de la prueba, reiterando que "cada familia o persona es libre de aceptar la toma de muestra de sangre".

Surgió en la asamblea la inquietud por la eliminación de la parte roja de la sangre una vez obtenido el suero; los tule (kuna) señalaron que no sabían cómo eliminarla, pues era la primera vez que esto ocurría; sabiendo que después de un lereo, los elementos utilizados se entierran en la casa donde se ejecuta el ritual, el equipo investigador propuso enterrar la sangre en la casa comunal; esto despertó zozobra en la comunidad, e inmediatamente los sailas y botánicos respondieron: "¿cómo se les ocurre decir que van a enterrar nuestras purbas (alma) si nosotros estamos vivos? Finalmente, la comunidad decidió que analizaría la cuestión y nos informaría.

En esta salida de campo obtuvimos 60 muestras de sangre, previa lectura del consentimiento informado, pues cada persona tenía derecho a negarse, a pesar de haber consentido a las evaluaciones anteriores.

Para solucionar la eliminación de los glóbulos rojos, se encomendó a uno de los botánicos más prestigiosos de la comunidad. El botánico dijo: "hemos analizado y la sangre debe ser llevada al río, porque el río es vida y da vida y la sangre del tule es vida, por lo tanto, la sangre se lleva al río"; de esta manera, el botánico acompañó la eliminación de los 60 tubos de sangre y se cercioró de que nosotros no la sacáramos de la comunidad.

En el siguiente período de trabajo de campo para continuar con la extracción de sangre nos encontramos con la siguiente posición: "el botánico que acompañó la eliminación de sangre dice que es suficiente, que sacaron mucha sangre, que ya deben parar, que es suficiente para ustedes estudiar". Tal como se había pensado en la formulación del proyecto, para la lógica tule, el número de 265 muestras no tenía razón de ser; se sentían muy expuestos a los riesgos implícitos en la pérdida de una parte de su duplo espiritual, la purba. Luego de mucho análisis se acordó nuevamente con la asamblea que quedaría a voluntad de cada persona aceptar la extracción de la sangre.

Meadows y colaboradores (11) plantean que los cambios inesperados por parte de las comunidades afectan el desarrollo de las investigaciones y generan conflictos en los investigadores, y señalan que se debe buscar un balance entre las tensiones y el respeto por los individuos y comunidades. Nuestro sentimiento inicial fue de angustia y de frustración, pensamos en nuestros objetivos y acuerdos, y sentimos que 
la comunidad estaba faltando a un compromiso adquirido, pero las consideraciones debidas a la ética nos llevaron a adoptar una posición diferente en los siguientes términos: comprendimos que los tule son interlocutores válidos, y que la fuerza de sus argumentos al señalar que se había extraído mucha purba (alma) y que ello los ponía en una situación de riesgo, debía respetarse. La relación de horizontalidad (28) establecida en el desarrollo de la investigación, en la cual cada sujeto participante constituía un interlocutor válido, y la reflexión se daba en un continuo entre el sujeto que participa y el sujeto investigador.Y, finalmente, el argumento de que "una norma es correcta cuando nadie la impone por la fuerza a los demás, si no que tras el proceso de deliberación todos pueden hacerla suya por el puro valor de los argumentos o las razones que la sustentan" (29).

Estas reflexiones se antepusieron a nuestros sentimientos iniciales y nos llevaron a darle prelación al bien de los tule (kuna) y al respeto por la decisión del colectivo, o sea, a acoger la recomendación de Meadows (11) de buscar el balance entre las tensiones y las decisiones de las comunidades.

La reflexión sobre la ética de la convicción y la ética de las consecuencias, dadas por Weber y retomadas por Camps (29) y Gracia (30), apoyan nuestra decisión de respeto a las razones dadas por los indígenas. La ética de la convicción significa ser fiel a los principios, y la ética de las consecuencias se refiere a la responsabilidad que se debe asumir por los actos propios, y significa que debe haber siempre una mediación entre nuestros principios y las consecuencias de nuestras acciones, so pena de caer en el fundamentalismo.

En este sentido, nuestro compromiso como investigadores era dar cuenta de los objetivos propuestos ante las entidades que apoyaban la propuesta investigativa, y ante la ciencia de la producción de conocimiento; sin embargo, en esta reflexión concluimos que las consecuencias de imponer la extracción de sangre serían lesivas para la cultura local, y que teníamos que preferir el bien de los tule (kuna) y el respeto a la unicidad de este colectivo frente a nuestro objetivo de investigación; por tanto, no entramos en engaños ni en artificios falsos para adquirir las demás muestras de sangre, simplemente conversamos con las personas y se extrajo la sangre a aquellas que lo permitieron. Finalmente, sólo se obtuvieron 80 muestras.

En cuanto a la investigación cualitativa, se resaltan a continuación cuatro aspectos que se tuvieron en cuenta durante el desarrollo de la investigación.

Con el método etnográfico, el investigador entra en la vida privada de la colectividad, la familia y el individuo. El principio bioético de respeto a las personas debe considerarse con rigor exhaustivo: respeto a la individualidad, a la privacidad, a la autonomía, a la intimidad, a la confidencialidad y a la unicidad o totalidad.

Los límites entre el respeto a la vida privada de las personas y el acopio de los datos de investigación se tornan borrosos si los investigadores no tienen claros los principios y valores morales, y si, además, no hay claridad sobre la competencia profesional centrada en la ciencia, porque "sin pensamiento crítico no hay ciencia, pues ésta tiene por misión hacer del pensamiento científico un método para construirse a sí misma [...]. La razón científica es inseparable de la razón ética" (31). Significa, entonces, que debe establecerse un equilibrio entre la recolección de datos etnográficos, el pensamiento crítico y las consecuencias, sin quedarse en una posición contemplativa de la cultura y sin emitir juicios o expresiones que laceren la dignidad humana de los integrantes del grupo estudiado.

Debe mantenerse el anonimato de los participantes, incluso entre ellos mismos: en el transcurso de la investigación ocurrió que al tratar en profundidad la temática, se preguntó a un participante sobre una cuestión específica, la cual hasta el momento estaba "parcialmente consentida"; inmediatamente los indígenas preguntaron: ¿Usted por qué sabe tanto? ¿Quién le dijo eso? Algunas veces hubo presión por saber quién nos había informado sobre el tema en el que queríamos profundizar. Ocurrió también el caso de un médico tule (kuna), muy reconocido 
en la comunidad, quien nos buscó y nos dijo: "Yo quiero dar a ustedes entrevista"; en un comienzo nos pareció muy extraño su ofrecimiento, pero le hicimos la entrevista. Posteriormente comprendimos que el rumor en estas comunidades pequeñas viaja muy rápido, y que lo que estaba en juego era el prestigio y la demostración de conocimiento entre ellos mismos.

Nuestra postura fue la de guardar el anonimato de las personas participantes con el fin de evitar confrontaciones, rivalidades, o luchas por el poder o el conocimiento; es decir, se buscó la no maleficencia entre los mismos participantes y el respeto a cada persona como interlocutor válido.

Hemos hablado de una relación de horizontalidad establecida de sujeto a sujeto. Así, el investigador indaga en el tule y el tule indaga en el investigador sobre su vida privada. Pero, además, en esta horizontalidad, el tule (kuna) vigilaba los límites de la información que se estaba obteniendo y que ellos "debían controlar" por temor a exponer por fuera de la comunidad la intimidad de su cultura. ¿Hasta dónde llevar la recolección de los datos? ¿Dónde suspenderla? ¿Qué publicar? Frente a estas preguntas entra a desempeñar un papel la mediación entre la ética de la convicción y la ética de la responsabilidad. Los tiempos han cambiado y las colectividades indígenas o individuos exigen al etnógrafo no exponer al desnudo ante los otros asuntos profundos, religiosos o íntimos de sus culturas.

En cuanto al daño que puede causarse a los participantes durante la recolección de la información, Cowles (32) señala que en las entrevistas algunos temas pueden estimular respuestas emocionales en los participantes, como el llanto, por ejemplo, ante lo cual el investigador debe estar preparado para suspender la entrevista, hacer intervención terapéutica o remitir a un terapeuta específico.

En el caso de los tule, el daño se refiere al efecto mágico de hablar de la enfermedad, pues quien habla de ella se expone a padecerla; teniendo en cuenta este referente, y para evitar el peligro que representa para el tule, hubo que ser discreto en el abordaje de las enfermedades, especialmente en las catalogadas como de mayor peligro para el colectivo, por ejemplo, las que tienen que ver con pérdida de sangre; en estos casos se intentó transferir el efecto del kuna hacia nosotros, es decir, para hablar de enfermedades referidas con la sangre, nos colocamos nosotros como sujetos que recibíamos la acción, así: si yo tengo diarrea con sangre y me siento mal, ¿usted como médico qué me haría?; si yo pasé la malaria y quedé pálida y débil, ¿usted qué tratamiento me haría?; de esta manera se minimizaron los riesgos de los tule al abordar estas temáticas, buscando la no maleficencia del colectivo.

\section{Reflexión final}

Cuando se trabaja con comunidades indígenas nos enfrentamos a culturas con valores morales diferentes a los nuestros. En estos encuentros culturales surgen diferentes dilemas éticos, tal como lo hemos analizado en esta experiencia investigativa, para los cuales la academia sólo da unas pinceladas, pues en nuestro medio la rigurosidad en la aplicación de los principios éticos ha sido más clara para los proyectos clínicos experimentales; los demás proyectos epidemiológicos no experimentales y los de corte cualitativo pasan a un segundo plano en lo que toca a las exigencias éticas.

Pensamos que todo proyecto investigativo con sujetos humanos requiere rigurosidad ética y vigilancia por parte de los comités de ética de las diferentes instituciones, tanto en la elaboración del proyecto como en su desarrollo, puesto que los asuntos éticos evolucionan con el tiempo y están en permanente cambio, dependiendo de las relaciones del investigador con los participantes (33) y de los procedimientos del proceso investigativo.

Colombia cuenta con cerca de 800.000 indígenas de amplia diversidad cultural: 84 grupos étnicos (34); sin embargo, carecemos de comités nacionales y locales de ética que regulen y vigilen el respeto, la justicia y la beneficencia de los individuos y comunidades durante el desarrollo de las investigaciones. Hasta el momento, en Colombia, los investigadores nacionales 0 extranjeros estamos solos y dirimimos los dilemas éticos que surgen con base en nuestros propios valores morales. 
En una sociedad existen diferentes culturas, y el modelo biomédico corresponde a un modelo cultural diferente al de las medicinas tradicionales (35); por tanto, cuando se trabaja con grupos étnicos (lo que no se refiere exclusivamente a indígenas), es importante que los investigadores conozcan su cultura, con el fin de tener elementos que les permitan tomar decisiones éticas en un marco de respeto frente a su diversidad.

Sin embargo, el conocimiento previo de la cultura del otro no significa que desde la elaboración de los proyectos se puedan dirimir todos los dilemas éticos, más aún cuando se trata de proyectos con extracción de fluidos corporales, puesto que las decisiones de los grupos varían según las tensiones políticas, económicas o sociales, lo que pone de manifiesto comportamientos inesperados para los investigadores. Es el caso de nuestra experiencia y también de la relatada por Meadows et al. (11). Significa entonces que en los proyectos de corte cualitativo, o que tengan que ver con muestras biológicas, los investigadores y las entidades financiadoras deben tener absoluta comprensión de las dificultades que eventualmente se presenten, ya que las culturas no son estáticas, y responden de manera diversa según el contexto.

Es urgente insistir sobre la necesidad de establecer comités de ética para asegurar los principios básicos de la ética en el trabajo con grupos étnicos, de manera que se preserve la vida, la cultura y el respeto a la diferencia. Dicha necesidad se acentúa con las políticas globales de comercio y salud. Es evidente la falta de una comisión de ética que oriente, vigile y regule a los investigadores en el acercamiento al conocimiento ancestral y los productos que de él se derivan.

Según la literatura, existe una relación directa entre hipovitaminosis $A$, desnutrición y malaria. EI objetivo principal de nuestro proyecto era buscar dicha relación; no obstante, por las razones descritas no fue posible este análisis. Empero, hubo hallazgos muy importantes, hasta el momento desconocidos, tanto para la propia comunidad kuna como para las entidades de salud estatal: la malaria supera más de cinco veces a la reportada en la región; la desnutrición en la población adulta es alarmante; el consumo de beta-caroteno es muy bajo, y en las 80 muestras de sangre, la hipovitaminosis A es alta (los datos serán presentados en otros artículos). A partir de estos hallazgos, la propia comunidad kuna ha intensificado las discusiones sobre salud y alimentación y la necesidad de rescatar alimentos tradicionales ricos en beta-caroteno, y las direcciones locales de salud de los municipios de Turbo y Necoclí están reforzando la vigilancia de la malaria.

Según Hurtado $(36,37)$, en la investigación con comunidades indígenas están involucrados factores muy complejos cuya comprensión y manejo sólo puede conseguirse con mayor número de investigaciones. Las dificultades que se presentan en el desarrollo de proyectos de este tipo no pueden desembocar en la disminución de los proyectos investigativos; al contrario, la investigación en salud debe continuar, teniendo presente los principios éticos de respeto, de justicia y de beneficencia por las comunidades indígenas y sus culturas.

Dejamos abierta la discusión sobre la importancia de pasar de un consentimiento informado, firmado por el participante al iniciar el proyecto, con el cual los investigadores salvan su responsabilidad, a un consentimiento en proceso, en el que cada fase de la investigación estará acompañada de la lectura y discusión del consentimiento con las personas involucradas, permitiendo el cese de su participación si así lo estiman conveniente, de manera que el proceso investigativo asuma en forma clara la reflexión sobre la aplicación de los principios éticos de justicia, de respeto a la unicidad de las personas y de las culturas.

En el desarrollo de las investigaciones surgen múltiples dilemas éticos que en ningún momento se contemplan en toda su dimensión al escribir la propuesta investigativa. El respeto a la dignidad humana, los principios y valores morales de los investigadores, la responsabilidad y las competencias profesionales son las herramientas para dirimir cualquier conflicto en favor del colectivo estudiado, sin negar la integridad de los investigadores. 
Estos dilemas, cuyos límites oscilan entre lo "borroso y lo claro" requieren, por tanto, del juicio crítico de un investigador apoyado en sus principios, de los valores morales y de sopesar las consecuencias para el sujeto o la colectividad estudiada, y abren el camino para la toma de decisiones éticas durante el proceso investigativo.

\section{Agradecimientos}

Al Instituto Colombiano para el Desarrollo de la Ciencia y la Tecnología "Francisco José de Caldas"- Colciencias y a la Universidad de Antioquia; a la Organización Indígena de Antioquia; a los líderes tules y a las personas de la comunidad por su participación y colaboración; a los directivos y al personal de salud de los hospitales y direcciones locales de salud de Necoclí y Turbo.

\section{Conflicto de intereses}

Las autoras manifiestan que no existe ningún conflicto de intereses.

\section{Financiación}

El estudio fue financiado por Colciencias y la Universidad de Antioquia. Código 1115-04-12962, Contrato RC. No. 517-2002.

\section{Referencias}

1. Vargas P. Los embera, los waunana y los cuna. En: Leyva P, editor. Colombia Pacífico. Tomo I. Bogotá: Fondo FEN Colombia; 1993. p.200-309.

2. Herrera Z. Grupos aborígenes de Panamá. 1997 [Consultado 2005, mayo 18]. Disponible en http:// www.monografias.com/trabajos14/indigenas-panama/ indigenas-panama.shtml.

3. Morales J. Cuna. En: Correa F, editor. Introducción a la Colombia amerindia. Bogotá: Instituto Colombiano de Antropología; 1987. p.263-77.

4. Meléndez I. Aspectos culturales relacionados con la salud y el ciclo vital femenino kuna. Rev Investigación y Educación en Enfermería 1992;10:131-51.

5. Morales J. Fauna. Trabajo y enfermedad entre los cuna. En: Correa F, editor. La selva humanizada: ecología alternativa en el trópico húmedo colombiano. Santa Fé de Bogotá: Instituto Colombiano de Antropología, Fondo FEN Colombia, Fondo Editorial CEREC; 1990. p.167-87.

6. Alcaraz G, Galeano A. El estatus de la mujer indígena kuna de Antioquia y su relación con el comportamiento reproductivo (tesis) . Medellín: Universidad de Antioquia; 1996.
7. Carmona-Fonseca J. La malaria en Colombia, Antioquia y las zonas de Urabá y bajo Cauca: panorama para interpretar la falla terapéutica antimalárica. latreia 2003;16:299-318.

8. Carmona-Fonseca J. La malaria en Colombia, Antioquia y las zonas de Urabá y bajo Cauca: panorama para interpretar la respuesta terapéutica antimalárica. latreia 2004;17:34-53.

9. Dirección Seccional de Salud de Antioquia._Eventos de vigilancia epidemiológica, ETV y ambiente. Antioquia, 1994-2003. Dirección Seccional de Salud de Antioquia. [Consultado 2005, julio 20]. Disponible en: www.dssa.gov.co/vectores/vectores.htm.

10. Organización Panamericana de la Salud/ Organización Mundial de la Salud. Normas éticas internacionales para las investigaciones biomédicas con sujetos humanos. Pub Cient 563. Washington: OPS; 1996.

11. Meadows LM, Lagendyk LE, Thurston WE, Eisener AC. Balancing culture, ethics and methods in qualitative health research with aboriginal peoples. International Journal of Qualitative Methods 2003;2. Disponible en: http://www.ualberta.ca/ iiqm/backissues/2_4/pdf/ meadows.pdf.

12. Alcaraz GM, Salazar AL, Huertas E, Agudelo JI. Epidemiología del cólera en Antioquia. Atención a comunidades indígenas. Boletín Epidemiológico de Antioquia, Servicio Seccional de Salud 1992;17;35-48.

13. Correa A. La malaria en los embera del Atrato medio antioqueño: una doble lectura (tesis). Medellín: Universidad de Antioquia. 1999. p.260.

14. Colombero G. La enfermedad, tiempo para la valentía. Santafé de Bogotá: San Pablo; 1993. p.101-17.

15. Fry S. La ética en la práctica de la enfermera: guía para la toma de decisiones éticas. Ginebra: Consejo Internacional de Enfermería; 1994. p.5-18, 49-56, 95.

16. Londoño JL, Alvarado EJ, Casas JV, Roselli DA. Normas científicas, técnicas y administrativas para la investigación en salud. Resolución No. 008430 de 1993. Santa Fe de Bogotá, D.C.: Ministerio de Salud; 1993.

17. Hammersley M, Atkinson P. Etnografía. Métodos de investigación. 2 ed. Barcelona: Paidós; 2001. p.15-37, 299.

18. Holmes W, Stewart P, Garrow A, Anderson I, Thorpe L. Researching aboriginal health: experience from a study of urban young people's health and well-being. Soc Sci Med 2002;54:1267-79.

19. Beuchamp T, Childress J. Principios de ética biomédica. Barcelona: Masson S.A.; 1999. p.113-326.

20. Arroyo MP, Cortina A, Torralba MJ, Zugasti J. Ética y legislación en enfermería. Análisis sobre la responsabilidad profesional. España: McGraw-Hill Interamericana; 1998. p.30-2. 
21. Davis A. Dilemas éticos y la práctica de enfermería. Perspectivas sobre la ética: principios morales. New York: Appleton, Century Crofts; 1983.

22. Torralba F. Constructos éticos del cuidar. Enferm Intensiva 2000;11:136-41.

23. Duque JH. El consentimiento informado en la práctica médica. Medellín: Hospital Universitario San Vicente de Paúl; 2001. p.13-31.

24. Munhall P. Ethical consideration in qualitative research. West J Nurs Res 1988;10:150-61.

25. Freeman WL. The protection of potencial individual volunteers and tribal communities in research involving the indian health service. IRB Appendix: 77-78. Protection of potential individual volunteers and tribal communities. [Consultado 2005, septiembre 9]. Disponible en: www.npaihb.org/epi/irb Protections_fr.pdf

26. Nordenskiold E. An historical and ethnological survey of the cuna indians. Suecia: Goteborg Museum, etnografiska, comparative etnographical studier 10; 1938. p.333-68.

27. Douglas M. Pureza e Perigo. São Paulo: Perspectiva; 1976. p.141-58.

28. Gracia D. De la bioética clínica a la bioética global. Treinta años de evolución. Revista Selecciones de Bioética 2004;6:4-7.

29. Camps V. Ética, retórica, política. Madrid: Alianza; 1995. p.97-8.
30. Gracia D. Fundamentación y enseñanza de la bioética. Santafé de Bogotá: Búho; 1998. p.124-6.

31. González MA. Aspectos éticos de la investigación cualitativa. Revista Iberoamericana de Educación 2002;29:90-1.

32. Cowles KV. Issues in qualitative research on sensitive topics. West J Nurs Res 1988;10:163-79.

33. Lipson J. Asuntos éticos en la etnografía. En: Morse $\mathrm{JM}$, editor. Asuntos críticos en los métodos de investigación cualitativa. Medellín: Universidad de Antioquia; 2003. p.389-415.

34. Lozano A. Cultivos de mata de coca en los resguardos indígenas. Etnias de Colombia. Medicina tradicional. [Consultado 2005, septiembre 9]. Disponible en: http:// www.etniasdecolombia.org/medicina.asp.

35. Kleinman A. Patients and healers in the context of culture: an exploration of the borderland between Anthropology, Medicine, and Psychiatry. California: University of California Press; 1980. p.24-70.

36. Hurtado AM, Hill KR. La salud comprometida de los indígenas suramericanos: necesidad de su estudio bajo normas éticas. Interciencia, Revista de Ciencia y Tecnología de América 2001;26:166-9.

37. Salzano FM, Hurtado AM. Lost paradises and the ethics of research and publication. New York: Oxford University Press; 2003. p.238. 